\title{
Tuberous sclerosis: unusual associations in four cases
}

\author{
EB ILGREN, ${ }^{*}$ D WESTMORELAND $\dagger$ \\ From the *Department of Neuropathology, Radcliffe Infirmary, Oxford, and †John Radcliffe Hospital, \\ Headington, Oxford
}

SUMMARY Tuberous sclerosis is a rare disease with classic primary or secondary changes affecting mainly the cerebrum, skin, kidneys, and heart. Such lesions are generally hamartomatous and thus display malignant features only in rare cases. This paper describes four cases of tuberous sclerosis which were unique in their association with certain unusual congenital, metabolic, and tumorous conditions.

Gomez ${ }^{1}$ recently reiewed 160 cases of tuberous sclerosis seen at the Mayo Clinic between 1935 and 1964. He defined both primary and secondary features of the condition. The primary features included lesions not only in the central nervous system such as cortical and subependymal tubers and retinal hamartomas, but also in other systems, such as the skin, including cutaneous angiofibromas and sebaceous adenomas. Secondary tuberous sclerosis lesions were considered to be shagreen patches, multiple renal tumours, and cardiac rhabdomyomas. In addition to these classic lesions, a multitude of other associated abnormalities have been described in tuberous sclerosis. ${ }^{1}$ We describe here four patients with tuberous sclerosis in whom several of the associated lesions were congenital, metabolic, and tumorous conditions that have not hitherto been described.

\section{Case histories}

\section{MATERIAL AND METHODS}

The diagnostic indices of the Department of Histopathology of the John Radcliffe Hospital, Oxford, were searched over the years 1935 to 1970 for cases of tuberous sclerosis studied at necropsy. Histological slides from each of these cases were recut when necessary and stained with haematoxylin and eosin, haematoxylin and van Gieson (HVG), phosphotungstic acid haematoxylin (PTAH) and periodic acid Schiff.

\section{RESULTS}

Nine cases of tuberous sclerosis with postmortem findings were found, and four of these, because of their unusual features, are described in detail.

Accepted for publication 16 November 1983
CASE 1 (21 year old woman)

\section{Clinical history}

At one year of age adenoma sebaceum was diagnosed. Five years later she developed epileptic fits and an electroencephalogram showed diffuse slow wave activity. At 17 years of age she developed a right spontaneous pneumothorax, which was treated by talc pleurodesis. Bullae were seen at operation. One year later she developed left sided abdominal pain and was found to have bilateral "polycystic" kidneys. Shortly thereafter she developed a left sided spontaneous pneumothorax, which was treated by a parietal pleurectomy. Once again bullae were seen at operation.

At the age of 20 she was found to be hypercalcaemic with no reduction in calcium concentrations during a standard hydrocortisone suppression test. The patient also had a reduced creatinine clearance. Nephrotomograms showed multiple lucent areas within both kidneys. Bone radiographs were negative for osteitis fibrosa, although cystic changes were seen in the terminal phalanges-changes consistent with tuberous sclerosis lesions. Her blood pressure was $150 / 95 \mathrm{~mm} \mathrm{Hg}$. Chest $x$ ray examination showed "honeycomb" changes in both lung fields and her pulmonary function tests were abnormal (DCO $10 \cdot 3$, predictive $31 \cdot 3$ ). Other investigations were normal: these included haemoglobin concentration; white blood cell count; erythrocyte sedimentation rate; blood urea, glucose, thyroxin, and plasmaprotein concentrations; electroencephalogram; electrocardiogram; and skull $x$ ray examination. Multiple filling defects were found on the liver scan.

A year later the patient underwent parathyroidectomy, and four hyperplastic parathyroid glands were found (histology showed chief cell hyperplasia). Postoperatively she was treated with dihydrotachy- 
sterol, and six days after operation was normocalcaemic and several months later normotensive. At 22 years of age the patient developed haemoptysis and one year later developed paroxysmal nocturnal dyspnoea due to cor pulmonale. In addition, she had abdominal pain, tachycardia (140 beats/min), and congestive heart failure (jugular venous pressure raised about $5 \mathrm{~cm}$ ) and was also found to be thyrotoxic. Shortly before her death right and left ventricular heaves and a third heart sound with vesicular breath sounds within the apices of both lungs were noted on examination. The day before she died the patient developed sepsis. Laboratory investigations at this time showed the following: haemoglobin $9 \mathrm{~g} / \mathrm{dl}$, a raised erythrocyte sedimentation rate ( $28 \mathrm{~mm}$ in the first hour), calcium concentration $2.35 \mathrm{mmol} / \mathrm{l}$, normal liver function tests, a reduced serum iron concentration $(6 \mu \mathrm{g} / \mathrm{l}$, normal $13-32 \mu \mathrm{g} /$ $\mathrm{l})$, and a raised thyroxine concentration $(216 \mu \mathrm{g} / \mathrm{l}$, normal 75-145 $\mu \mathrm{g} / \mathrm{l})$. A blood film showed 260 leucocytes but none of them polymorphonuclear. Four blood cultures were negative. Lymphocytes accounted for $76 \%$ and monocytes for $20 \%$ of the leucocytes.

\section{Family history}

The patient's mother, who also had a slight degree of adenoma sebaceum, was found to have headaches at the age of 51 and also to be hypertensive and hypercalcaemic. Hyperparathyroidism was diagnosed and four hyperplastic parathyroid glands were removed.
Pathological findings

Nervous system. Although the brain was of normal size $(1250 \mathrm{~g})$, some gyri were abnormally small and firm with numerous cortical tubers. (Fig. 1 and 2) On section there were "candle gutterings" along the surfaces of both lateral veritricles. The brain stem and cerebellum showed areas of abnormal firmness predominantly in the white matter. Histologically, there were numerous malformed cortical layers (Fig. 3 ) in the cerebrum and these contained large abnormally shaped cells probably either neuronal or astrocytic in origin (Fig. 4). There was also calcification in small areas of the cerebrum and giant heterotopic neurones in the brain stem and cerebellum.

Heart. The heart showed right ventricular hypertrophy. There was also a rhabdomyoma within the apex of the left ventricle, and mural thrombi were found over the endocardium of both ventricles.

Skin. There were multiple areas of adenoma sebaceum over the skin of the nose and cheek and numerous subungual fibromata.

Liver. The liver was enlarged (2050 g) and contained six white nodules, the largest of which measured $6 \mathrm{~cm}$ in diameter. Microscopically, several of these were found to be angiomyolipomata.

Pancreas. There was a large islet cell tumour (Fig. 5) adjacent to the pancreas.

Lungs. Multiple small cysts and nodules around 1 $\mathrm{cm}$ in diameter were found throughout each lung. Histologically, the pulmonary lesions contained numerous macrophages and many small nodules of proliferating smooth muscle. In addition, the small

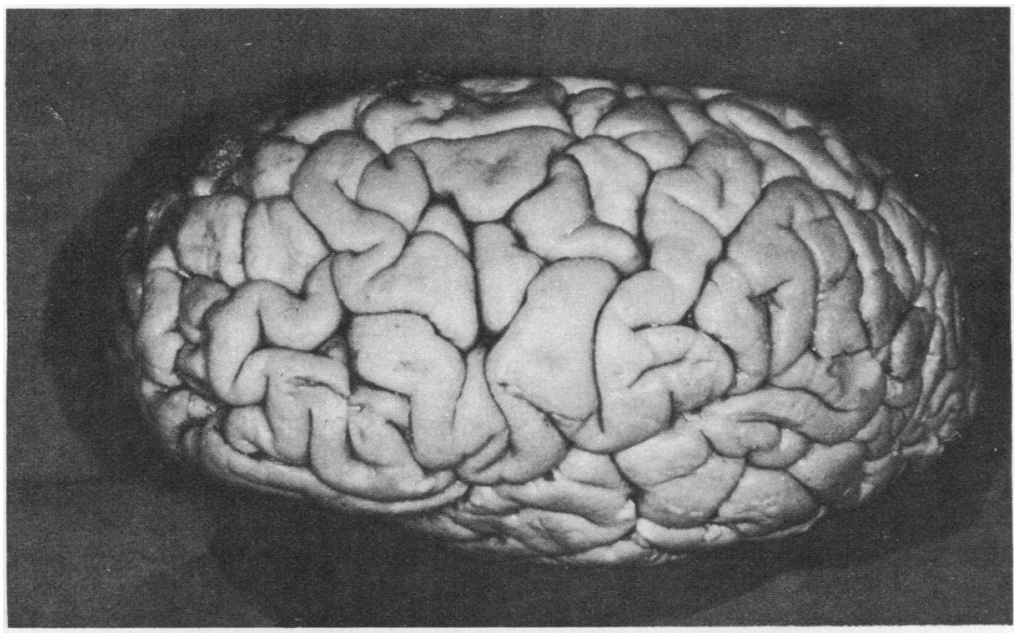

Fig. 1 Cerebral hemispheres (1-low power) showing typical cortical "tubers" with firm, raised, smooth, somewhat expanded, umbilicated gyral crests. 


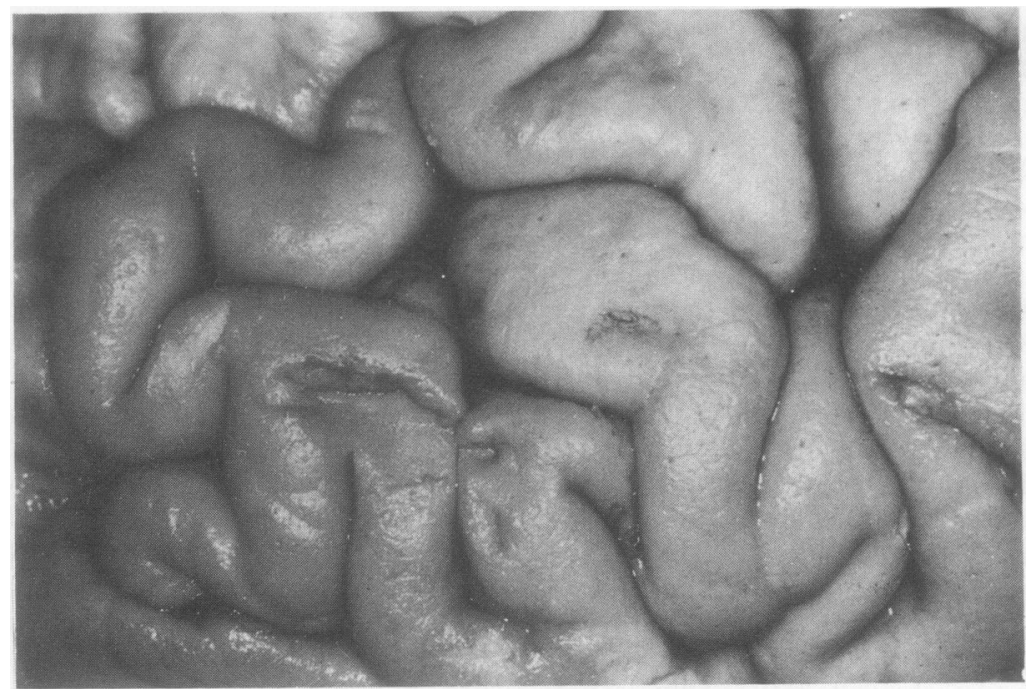

Fig. 2 Detail of Fig. 1.

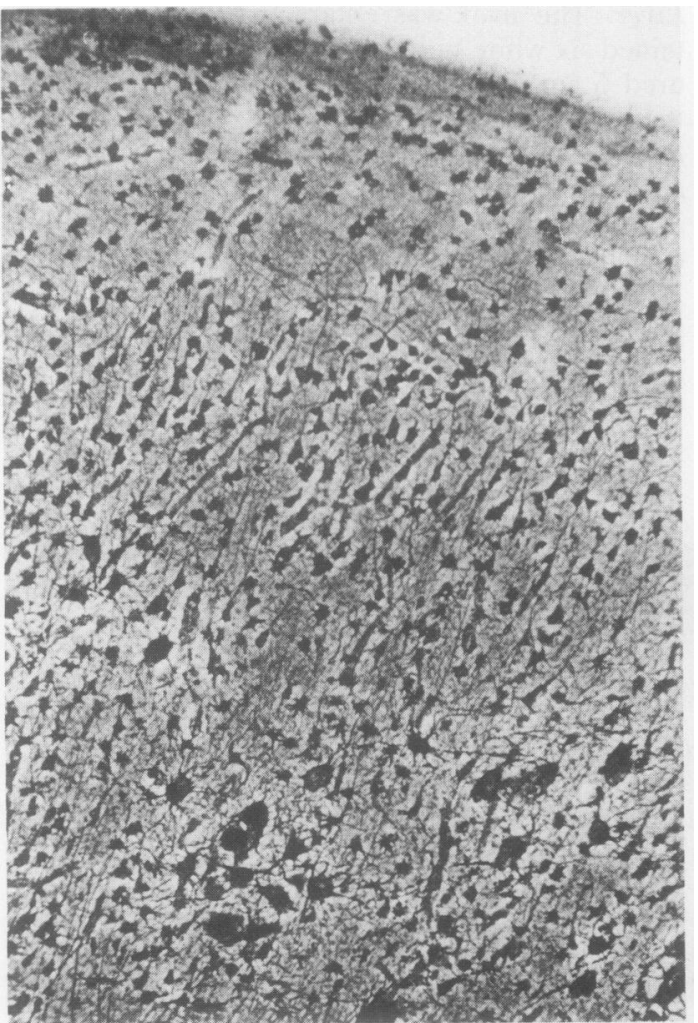

Fig. 3 Microscopic section of cortex showing moderate disorganisation of normal laminar pattern with occasional groups of very large cells either neuronal or astrocytic in character. Silver $\times 50$.

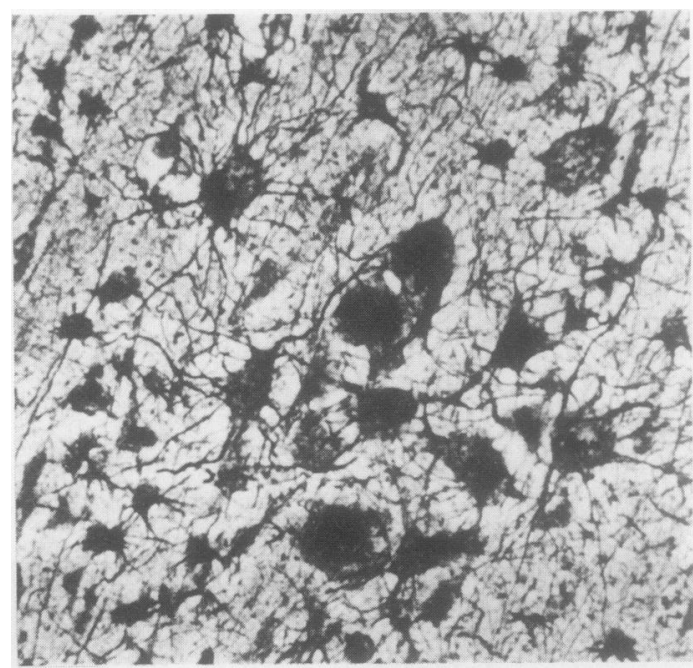

Fig. 4 Detail of Fig. 3. Silver $\times 200$.

pulmonary arterioles were rather hypertrophic.

Adrenal and pituitary glands. Adenomata were found in the right adrenal gland and in the pituitary gland.

Kidney and para-aortic lymph nodes. Both kidneys were considerably enlarged, the left kidney weighing $765 \mathrm{~g}(19 \times 12 \times 9 \mathrm{~cm})$ and the right kidney weighing $500 \mathrm{~g}(18 \times 9 \times 5 \mathrm{~cm})$. The right kidney appeared to be largely replaced by a focally haemorrhagic angiomyolipoma while the left kidney contained both angioliposarcoma as well as areas of adenocarcinoma (Fig. 6). The adjacent para-aortic 


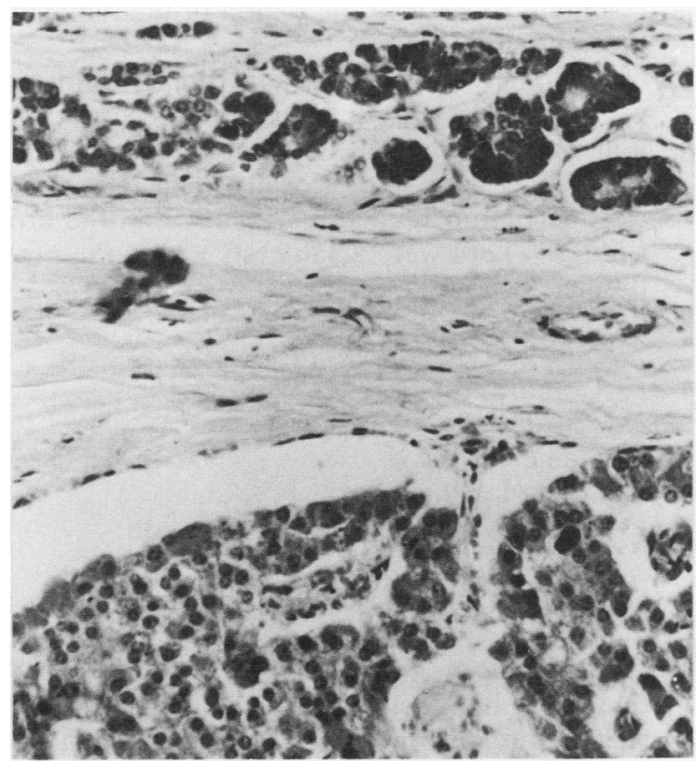

Fig. 5 Islet cell tumour of the pancreas showing compressed pancreatic acini (top) and tumour (below). Haematoxylin and eosin $\times 50$.
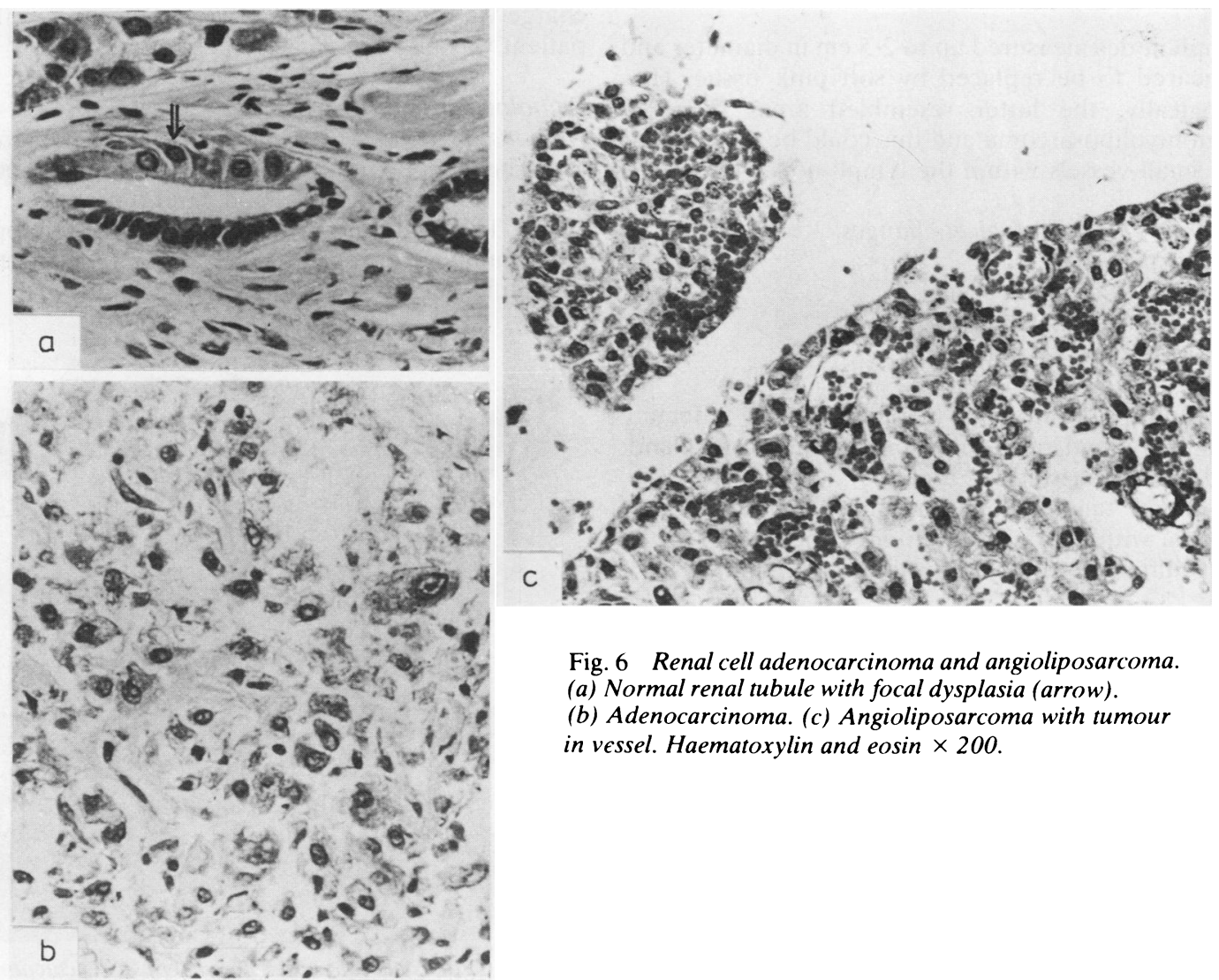

Fig. 6 Renal cell adenocarcinoma and angioliposarcoma. (a) Normal renal tubule with focal dysplasia (arrow).

(b) Adenocarcinoma. (c) Angioliposarcoma with tumour in vessel. Haematoxylin and eosin $\times 200$. 


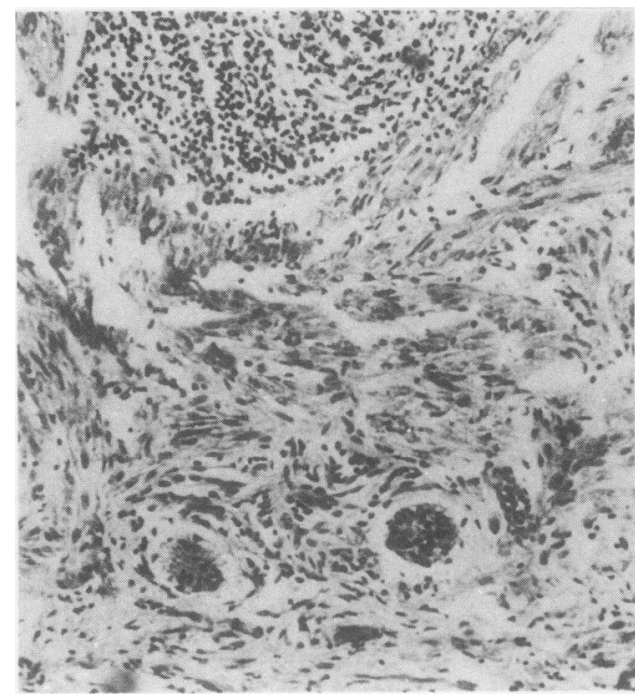

Fig. 7 Infiltration of para-aortic lymph nodes by angiomyoliposarcoma. Haematoxylin and eosin $\times 50$.

lymph nodes measured up to $2.5 \mathrm{~cm}$ in diameter and appeared to be replaced by soft pink tissue. Histologically, the latter resembled areas of renal angiomyoliposarcoma and this could be seen invading small vessels within the lymph node (Fig. 7).

\section{Summary of pathological changes:}

1 Cortical tubers

2 Cardiac rhabdomyoma

3 Subungual fibromas

4 Adenoma sebaceum

Associated with:

1 Multiple endocrine adenomatosis affecting pituitary gland, adrenal gland, parathyroid gland, and pancreas (islet cell tumour)

2 Renal angiomyoliposarcoma and adenocarcinoma with regional lymph node metastases

3 Pulmonary and hepatic angiomyolipomata

CASE 2 (6 week old boy)

\section{Clinical history}

The clinical history was lost, although it is known that the patient's mother and brother had tuberous sclerosis.

\section{Pathological findings}

Nervous system. The cerebrum showed multiple cortical nodules, which were prominent in the left temporal $(3 \mathrm{~cm}$ in diameter) and right frontal $(3 \mathrm{~cm}$ in diameter) lobes. There was also "candle guttering" along the surfaces of the lateral ventricles with sub- ependymal gliosis in the region of the thalamus and the caudate nucleus.

Heart. There was a rhabdomyoma within the left ventricular myocardium. An infantile aortic coarctation was also found.

Lungs. The lungs were focally atelectatic and there was meconium stained material within the bronchi. Kidney. There were small cysts within the proximal tubules of both kidneys.

\section{Summary of pathological lesions:}

1 Cerebral tubers

2 Cardiac rhabdomyoma

3 Renal cysts

Associated with:

1 Infantile type of aortic coarctation

CASE 13 (2 month old girl)

\section{Clinical history}

Only a limited history was available. The child was well before her admission except for a history of continued nasal discharge. The culture of the discharge was positive for Staphylococcus aureus. The patient was also hypercalcaemic before death.

\section{Pathological findings}

Nervous system. The brain contained numerous giant heterotopic neurones round the aqueduct of sylvius, lower brain stem, and cerebrum.

Heart. The heart showed biventricular hypertrophy and contained a fibromuscular tumour obstructing the outflow tract of the left ventricle.

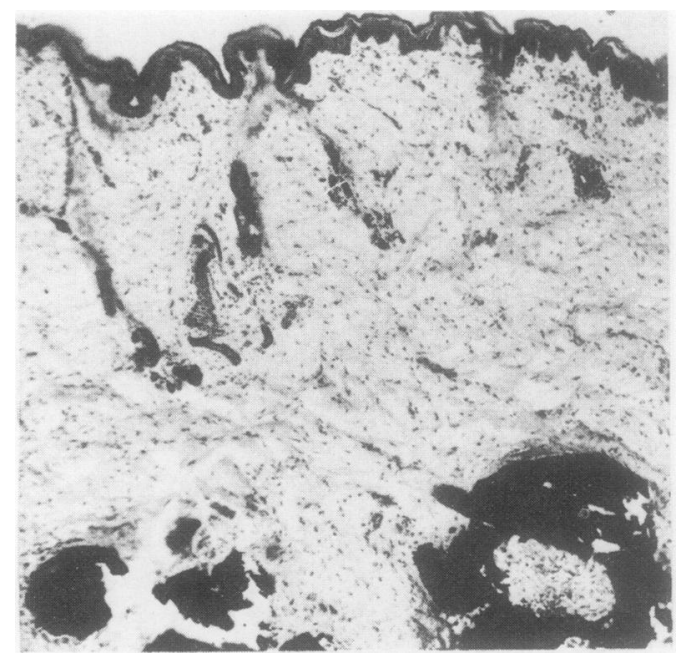

Fig. 8 Universalis calcinosis. Focal deposits of calcium can be seen in the subdural connective tissue. Von Kossa $\times 40$. 


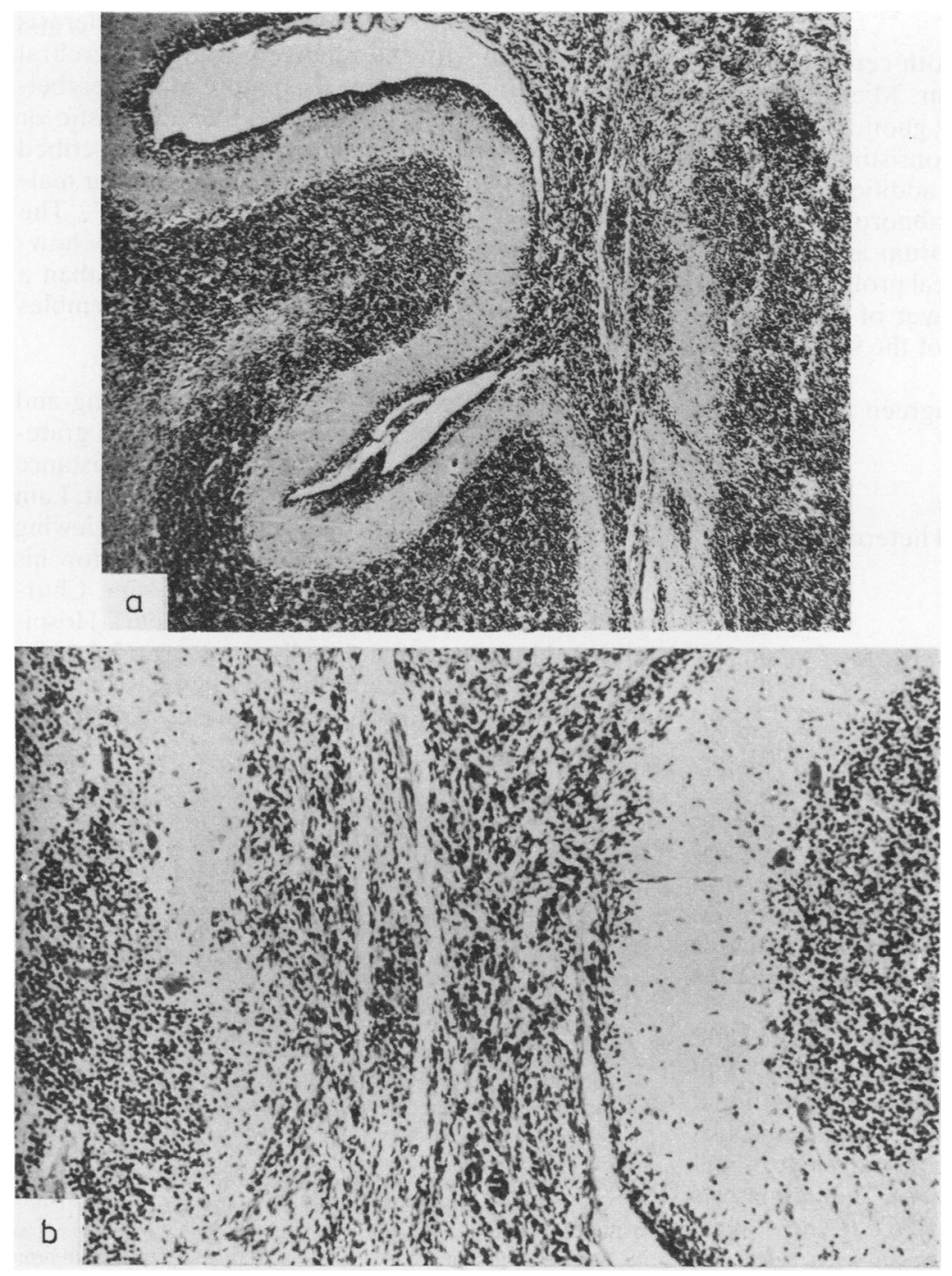

Fig. 9 Dysplasia and hyperplasia of cerebellar mantle layer. Haematoxylin and eosin. (a) Lower power $\times 40$. (b) High power $\times 150$.

Skin. There was a diffuse patchy pigmentation of the skin. Numerous subcutaneous nodules, plaques, and ridges were found particularly along the left side of the back, neck, and face, some of which were centrally ulcerated. Histologically, there was much focal subcutaneous calcification (Fig. 9) which resembled that seen in universalis calcinosis.

Lungs. The lungs were focally atelectatic with numerous areas of fibrosis and lymphocytic infiltration.

Summary of pathological lesions:

1 Neuronal heterotopias

2 Cardiac fibromyomas
Associated with:

1 Universalis calcinosis

2 Pulmonary fibrosis

CASE 4 ( 8 week old girl)

\section{Clinical history}

Only a limited history was available. The patient had a three week history of respiratory distress and postprandial vomiting before death. On examination she was found to be a thin small baby with spasticity of all four limbs and bilateral talipes, holding her arms in flexion and legs in extension. 
Pathological findings

Nervous system. At necropsy, both cerebral hemispheres were found to be nodular. Microscopically, these areas were considerably gliotic, containing neuronal heterotopias often consisting of ependymoblasts forming rosettes. In addition, focal cortical and subependymal glial abnormalities were seen in many parts of the cerebrum and the brain stem. There was considerable focal proliferation and disorganisation of the mantle layer of the cerebellum (Fig. 9) with local invasion of the subarachnoid space.

Skin. There were numerous shagreen patches over the face.

Summary of pathological lesions:

1 Cortical tubers with neuronal heterotopias of the cerebrum

2 Shagreen patches

Associated with:

1 Cerebellar dysplasia and possibly neoplasia (medulloblastoma)

\section{Discussion}

Tuberous sclerosis is relatively rare and occurs in about one in 100000 to 170000 people in the general population (see Table 13.4 in Kuntz and Gomez $^{2}$ ). Its infrequency is also strongly suggested by Gomez's study, ' in which he found only 160 cases in 1.5 million patients seen at the Mayo Clinic over 30 years.

Most cases of tuberous sclerosis recorded to date have had classic primary or secondary lesions.' The patients described in this report are exceptional since certain congenital, metabolic, and tumorous conditions were seen in association with their tuberous sclerosis. The multiple endocrine adenomatosis syndrome found in case 1 has not, to our knowledge, been described before in association with tuberous sclerosis, although Gutman ${ }^{3}$ recorded an islet cell tumour of the pancreas in patients with this disorder. Case 1 is even more unusual in its association with an angioliposarcoma ${ }^{4}$ and a renal cell carcinoma, ${ }^{56}$ since most renal tumours in tuberous sclerosis are benign. Case 2 is unusual in its association with aortic coarctation since only one such case has been described previously.' Universalis calcinosis, which was found in case 3, has not previously been seen in patients with tuberous sclerosis. Finally, in case 4 the finding of a pronounced proliferation of the cerebellar mantle layer is most striking. Cerebellar lesions, as described by Reagan, ${ }^{8}$ are seen infrequently in patients with tuberous sclerosis in comparison with the relatively common cerebral tubers. ${ }^{9}$ Moreover, the precise nature of the cerebellar changes seen in case 4 , whether dysplastic or neoplastic, remain speculative. Urich" described focal cerebellar dystopias ranging from " minor malformations to total cortical disorganisation". The subarachnoid space invasion found in case 4, however, is more likely to be neoplastic change than a dystopic or dysplastic alteration and thus resembles an "incipient medulloblastoma".

I am indebted to Mr Ron Beesley for cutting and staining many of the sections described. I am grateful to Mr John Hayward for photographic assistance and to Mrs G Ilott for typing of the manuscript. I am also grateful to Dr Michael Dunnill for reviewing the sections described in this paper and for his encouragement and to Dr RH Lindenbaum, Churchill Hospital, and Dr Jennifer Dennis, Park Hospital, for their comments. Finally, I am grateful to Dr Joan Rivett, Department of Pathology, Stoke Mandeville Hospital, for the postmortem report and blocks of case 1 .

\section{References}

' Gomez MR, ed. Clinical experience at the Mayo clinic. In: Tuberous sclerosis. New York: Raven Press, 1979:27-54.

${ }^{2}$ Kuntz NL, Gomez MR. Genetics, population studies, and pathogenesis. In: Tuberous sclerosis. New York: Raven Press, 1979: 207-20.

${ }^{3}$ Gutman A, Lephkowitz M. Tuberous sclerosis associated with spontaneous hypoglycaemia. $\mathrm{Br}$ Med J 1959;ii: 1065-8.

${ }^{4}$ Hartveit F, Harreaker B. A report of 3 angiolipomyomata and 1 angioliposarcoma. Acta Pathol Microbiol Scand 1960;49:329-6.

s Kavaney P, Fielding I. Angiomyolipoma and renal cell carcinoma in the same kidney. Neurology 1963;6:643-6.

- Berg J. Angiomyosarcoma of kidney (malignant hamartomatous angiomyolipoma) in a case with solitary metastasis from bronchogenic carcinoma. Cancer 1955;8:759-63.

'Rabinowicz T. Les tumeurs cerebrales chez l'homme. Bull Acad Suisse Sci Med 1968;24:144-67.

${ }^{8}$ Reagan TJ. Neuropathology. In: Tuberous sclerosis. New York: Raven Press, 1979:69-84.

- Van Bogaert L, Paillas JE, Berard-Badier M, Payan H. Etude sur la sclerose tubereuse de Bourneville a forme cerebelleuse. $R e v$ Neurol 1958;98:673-89.

${ }^{10}$ Urich $\mathrm{H}$. Malformations of the nervous system, perinatal damage, and related conditions in early life. In: Blackwood W, Corsellis J, McMinimy W, eds. Greenfield's neuropathology. London: Arnold Press, 1977:361-470.

Requests for reprints to: Dr EB Ilgren, Senior Registrar, Department of Neuropathology, Radcliffe Infirmary, Oxford OX2 6HE, England. 